\title{
Intravitreales Ranibizumab bei prädominant hämorrhagischer choroidaler Neovaskularisation im Rahmen der altersbedingten Makuladegeneration
}

\author{
Schlüsselwörter \\ Altersbedingte Makuladegeneration · Fundusbezogene \\ Mikroperimetrie · Ranibizumab intravitreal · Prädominant \\ hämorrhagische choroidale Neovaskularisation · Intravitreale \\ Injektionen
}

\footnotetext{
Zusammenfassung

Zielsetzung: Beurteilung der Auswirkungen der Monotherapie mit intravitrealem Ranibizumab bei prädominant hämorrhagischer choroidaler Neovaskularisation mit Beteiligung der Fovea im Rahmen der altersbedingten Makuladegeneration.

Material und Methoden: 22 Augen mit hämorrhagischer Neovaskularisation wurden konsekutiv mit 3 monatlichen intravitrealen Ranibizumab-Injektionen behandelt. Weitere Injektionen wurden innerhalb des 12-monatigen Nachbeobachtungszeitraums gemäß den Kriterien für die Wiederbehandlung verabreicht.

Ergebnisse: Im Mittel wurden 6,64 \pm 1,36 Injektionen verabreicht. Insgesamt verbesserte sich der mittlere Visus in 12 Monaten von 10,90 \pm 6,02 auf 12,81 \pm 8,34 ETDRS-Buchstaben (ETDRS = Early Treatment Diabetic Retinopathy Study; $p>0,05)$. Die «frühe Behandlungsgruppe» zeigte im Mittel eine Verbesserung um 2,83 \pm
}

2,24 ETDRS-Buchstaben ( $p<0,05)$, während die «späte Behandlungsgruppe» sich im Mittel um 0,30 \pm 1,25 ETDRS-Buchstaben verbesserte $(p>0,05)$; der Unterschied zwischen beiden Gruppen war signifikant $(p<0,05)$. Bei 20 Patienten war ein progressives Abklingen der Makulablutung zu beobachten (mittlere Dauer: 5,3 \pm 1,6 Monate).

Schlussfolgerungen: Ranibizumab-Injektionen können als nutzbringender Ansatz zur Behandlung der prädominant hämorrhagischen choroidalen Neovaskularisation mit fovealer Beteiligung im Rahmen der altersbedingten Makuladegeneration betrachtet werden. Hierbei scheint das Zeitintervall zwischen dem Auftreten der Blutung und der ersten Injektion ein wichtiger prädiktiver Faktor für das Endergebnis beim Visus zu sein.

(c) 2016 S. Karger GmbH, Freiburg

\section{Originalartikel}

\section{Intravitreal Ranibizumab for Predominantly Hemorrhagic Choroidal Neovascularization in Age-Related Macular Degeneration}

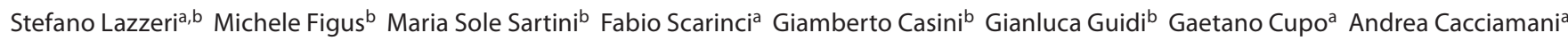
Vincenzo Fasanella ${ }^{e}$ Luca Agnifilie Paolo Piaggi ${ }^{c, d}$ Monica Varano ${ }^{a}$ Guido Ripandellia Marco Nardi ${ }^{\mathrm{b}}$ Mariacristina Parravano ${ }^{\mathrm{a}}$

aFondazione G.B. Bietti, IRCCS Rome, Rom, Italien;

${ }^{b}$ Ophthalmic Unit, Surgical, Medical and Molecular Pathology and Critical Area, University of Pisa, Pisa, Italien;

'Department of Endocrinology and Metabolism, University of Pisa, Pisa, Italien;

${ }^{d}$ Department of Energy and Systems Engineering, University of Pisa, Pisa, Italien;

eOphthalmic Clinic, Department of Medicine and Ageing Science, University of Chieti-Pescara,

Chieti, Italien 
Prof. Dr. Lars-Olof Hattenbach, FEBO

hattenbach.lo@klilu.de

\section{Transfer in die Praxis}

Die Therapie der exsudativen altersbedingten Makuladegeneration (AMD) durch die intravitreale Injektion von VEGF-Hemmstoffen (VEGF = vascular endothelial growth factor) wurde in den vergangenen Jahren in zahlreichen Studien mit Tausenden von Patienten eingehend untersucht und gilt heute als unumstrittener Standard der Behandlung. Weniger eindeutig ist indes die Vorgehensweise beim Auftreten subretinaler Blutungen, einer häufigen Komplikation bei AMD-bedingter choroidaler Neovaskularisation. Da diese Patientengruppe in vielen Studien aufgrund von Ausschlusskriterien fehlt und nie Ziel von großangelegten Untersuchungen zur Therapie der exsudativen AMD war, erweist sich die Datenlage zur Behandlung solcher Patienten als erstaunlich dünn. Die verfügbare Literatur beschränkt sich auf kleinere Fallserien oder Subgruppenanalysen größerer Studien, deren Design zur Beantwortung anderer Fragestellungen gedacht war.

Ein interessanter Ansatz, wie bei Patienten mit exsudativer AMD und subretinaler Blutung therapeutisch vorgegangen werden sollte, wurde nun in der hier kommentierten, kürzlich publizierten
Studie einer italienischen Arbeitsgruppe vorgestellt. Lazzeri et al. untersuchten den Effekt einer intravitrealen Therapie mit Ranibizumab bei Patienten mit AMD-bedingter hämorrhagischer choroidaler Neovaskularisation und Beteiligung der Fovea. Dabei erhielten die Patienten 3 monatliche Ranibizumab-Injektionen und eine anschließende Weiterbehandlung nach Bedarf über einen Verlauf von 12 Monaten. Doch nicht nur das: Lazzeri et al. untersuchten auch den Effekt einer frühzeitigen Therapie mit Ranibizumab, indem sie Patienten, bei denen eine Behandlung bereits innerhalb der 1. Woche nach Auftreten der Symptome erfolgt war, retrospektiv mit jenen verglichen, die erst nach Ablauf der 1. Woche bis zu einer maximalen Latenzzeit von 4 Wochen therapiert worden waren.

Das Ergebnis war eindeutig: Nach Ablauf der Nachbeobachtungszeit von 12 Monaten zeigte sich ein signifikanter Anstieg der mittleren Sehschärfe von 10,90 ( $\pm 6,02)$ auf 12,81 ( $\pm 8,34)$ ETDRS-Buchstaben (ETDRS = Early Treatment Diabetic Retinopathy Study). In der Gruppe der innerhalb 1 Woche nach Auftreten der Symptome

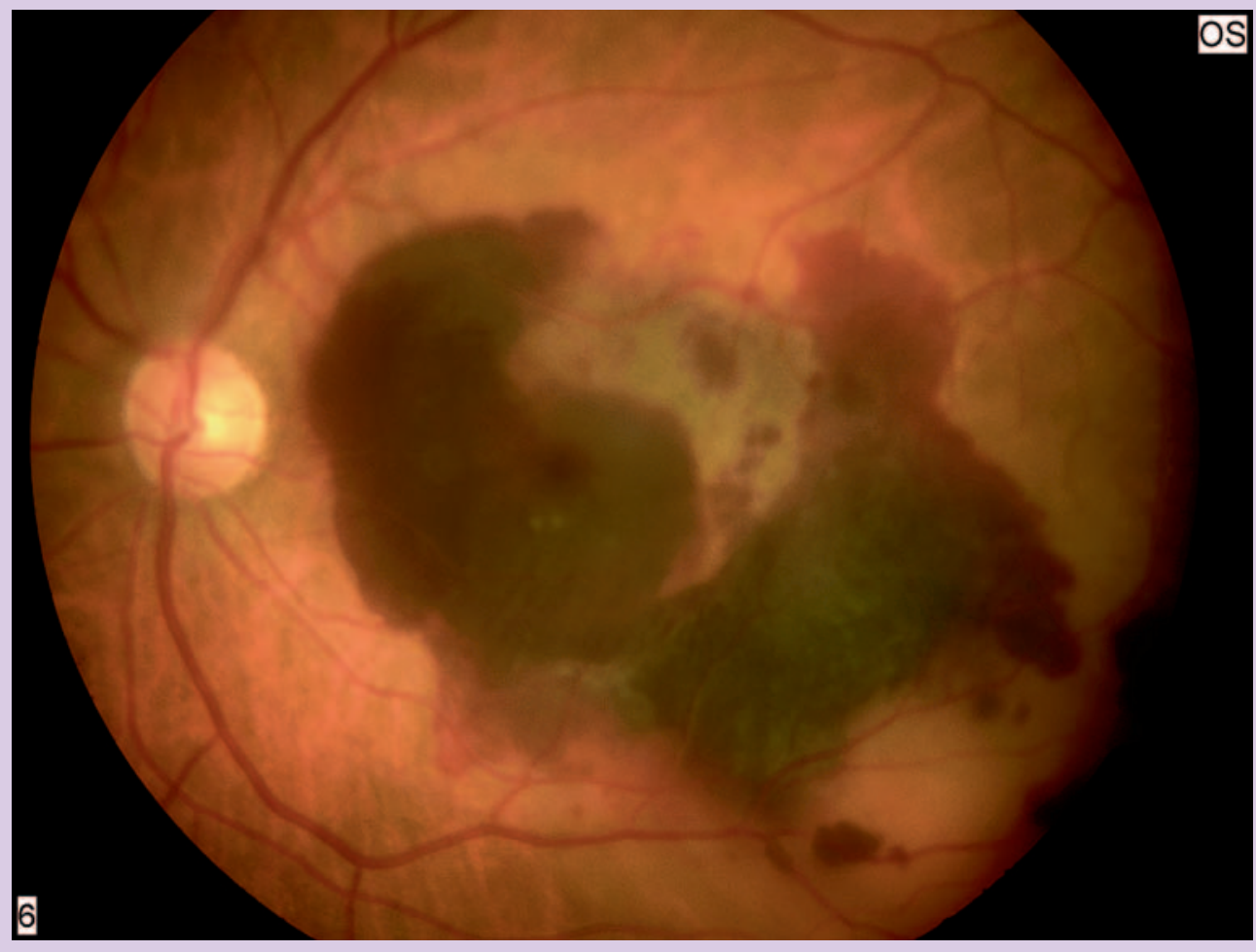

Abb. 1. Fundusbild einer subretinalen Blutung bei exsudativer AMD. Die hier vorgestellte Studie von Lazzeri et al. zeigt, dass in solchen Fällen eine frühzeitig durchgeführte Monotherapie mit intravitrealem Ranibizumab als Behandlungsoption durchaus infrage kommt. 
therapierten Patienten beobachteten die Autoren einen mittleren Visusanstieg um 2,83 ( $\pm 2,24)$ ETDRS-Buchstaben, während später Behandelte mit 0,30 $( \pm 1,25)$ ETDRS-Buchstaben eine signifikant schlechtere Visusentwicklung aufwiesen. Dabei war auch die durchschnittliche Anzahl an Injektionen in der frühen Behandlungsgruppe mit 5,57 $( \pm 1,15)$ signifikant geringer als in der Gruppe der spät behandelten Patienten mit 8,51 ( $\pm 1,72)$.

Bemerkenswert an den von Lazzeri et al. vorgestellten Daten ist auch, dass Patienten mit größeren Blutungen auf die geschilderte Art und Weise behandelt wurden. Nach einem Beobachtungszeitraum von 12 Monaten waren die mittlere Läsionsgröße von $21,1 \mathrm{~mm}^{2}$ auf 13,2 $\mathrm{mm}^{2}$ und die mittlere zentrale Netzhaut-Dicke

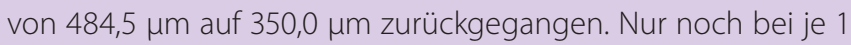
Patienten in jeder Behandlungsgruppe zeigte sich eine Blutung im Bereich der Makula, wobei es nur bei 7,1\% der früh Therapierten, aber 62,5\% der spät Behandelten zu einer submakulären Fibrose gekommen war. Interessant ist also auch die Beobachtung, dass eine frühzeitige intravitreale Anti-VEGF-Therapie offenbar einer Narbenbildung nach Blutung entgegenwirkt, denn genau dies galt bisher als Argument für eine invasivere Vorgehensweise. So finden sich insbesondere zur Behandlung großer subretinaler Blutungen bisher überwiegend solche Studien, die eine Vitrektomie und subretinale Drainage oder mechanische Verdrängung der Blutung durch eine Gastamponade postulieren, während Untersuchungen zum therapeutischen Effekt einer intravitrealen Monotherapie mit Anti-VEGF-Wirkstoffen im Wesentlichen auf die Behandlung kleiner Hämorrhagien beschränkt waren.

\section{Fazit}

Auch wenn die Ergebnisse der hier vorgestellten Studie in Anbetracht der geringen Fallzahl und des retrospektiven Charakters mit Vorsicht interpretiert werden müssen, so liefert diese von der Arbeitsgruppe um Lazzeri sehr sorgfältig und mit allen klinisch relevanten Parametern dokumentierte, aufmerksam analysierte Untersuchung doch stichhaltige Argumente dafür, dass Patienten mit hämorrhagischer exsudativer AMD durch eine frühzeitig durchgeführte Monotherapie mit Ranibizumab bzw. VEGF-Hemmern erfolgreich behandelt werden können. Eine invasivere Vorgehensweise ist in solchen Fällen also meist nicht zwingend erforderlich, wenngleich die chirurgische Intervention für subretinale Massenblutungen weiterhin die einzige Behandlungsoption bleiben dürfte.

Kontaktadresse: Prof. Dr. Lars-Olof Hattenbach, FEBO, Augenklinik des Klinikums Ludwigshafen, Bremserstraße 79, 67063 Ludwigshafen, Deutschland 